\title{
VIEWPOINTS
}

\section{VIEW: Is Drinking to Thirst Adequate to Appropriately Maintain Hydration Status During Prolonged Endurance Exercise? Yes}

\author{
Martin D. Hoffman, MD; James D. Cotter, PhD; \\ Éric D. Goulet, PhD; Paul B. Laursen, $\mathrm{PhD}$ \\ From the Department of Physical Medicine \& Rehabilitation \\ Department of Veterans Affairs, Northern California Health Care \\ System, and University of California Davis Medical Center, \\ Sacramento, CA, USA (Dr Hoffman); the Exercise and Environmental \\ Physiology, School of Physical Education, Sport and Exercise Sciences \\ Division of Sciences, University of Otago, Dunedin New Zealand \\ (Dr Cotter); the Research Centre on Aging, Faculty of Physical Activity \\ Sciences, University of Sherbrooke, Sherbrooke, QC Canada \\ (Dr Goulet); and the High Performance Sport New Zealand, and \\ Sports Performance Research Institute New Zealand (SPRINZ) \\ Auckland University of Technology, Auckland New Zealand \\ (Dr Laursen).
}

\section{Importance of the Question}

The importance of adequate fluid intake during exercise has been stressed for sport and cardiovascular performance, for management of thermal stress, ${ }^{1,2}$ and for prevention of exercise-induced muscle cramping ${ }^{1,2}$ and acute kidney injury from rhabdomyolysis. ${ }^{1}$ For these reasons, several recent guidelines recommend that mass loss during exercise should not exceed $2 \%$ of body mass. ${ }^{1,2}$ We will show why we believe that such recommendations are not well supported by ecologically valid science, and may even induce harm.

Exercise-associated hyponatremia (EAH) is a primary concern for fluid intake beyond that which is physiologically appropriate before, during, and after exercise. ${ }^{3}$ The behavior of overhydration typically occurs from misunderstandings about fluid needs during exercise,

The authors have no pertinent disclosures or conflicts of interest.

Corresponding author: Martin D. Hoffman, M.D., Department of Physical Medicine \& Rehabilitation (117) Sacramento VA Medical Center 10535 Hospital Way, Sacramento, CA 95655-1200; Phone: 916-843-9027; Fax: 916-843-7345; (e-mail: mdhoffman@ucdavis.edu). coupled with excessive concerns about dehydration and the need for adequate hydration to prevent decline in performance, heat illness, and muscle cramping, likely fueled by various organizational hydration guidelines. Because there have been several deaths, as well as other morbidity, from $\mathrm{EAH},{ }^{3}$ a discussion to clarify current knowledge about proper hydration during exercise extends beyond merely being of academic interest.

\section{Fluid Balance During Exercise}

Glycogen oxidation during exercise results in mass loss, the extent of which depends on the intensity and duration of the exercise. Associated with the oxidation of glycogen is release of water, as 1 to $3 \mathrm{~g}$ of water are stored with every gram of glycogen. ${ }^{4}$ Thus, it is important to recognize that a loss of body mass during moderate-tohigh-intensity exercise does not necessarily imply dehydration or a reduction in the cardiovascular fluid compartment, as is commonly inferred. In fact, total body water has been found to be maintained despite a loss in body mass of approximately $3.5 \%$ among runners participating in a $56-\mathrm{km}$ ultramarathon ${ }^{5}$ and a loss in body mass of approximately $2 \%$ among soldiers during a 14.6-km march. ${ }^{6}$ Furthermore, when well-trained cyclists lost $3 \%$ of body mass by performing 2 hours of submaximal exercise (walking and cycling) in the heat, and were then reinfused with saline in a blinded fashion so that they were $0 \%, 2 \%$, or $3 \%$ below their initial body mass before a $25-\mathrm{km}$ bike ergometer time trial in the heat, blood volume returned to baseline during the time trial irrespective of the extent of fluid restoration. ${ }^{7}$ Thus, consistent with conclusions made several decades ago, humans are well designed to resist volume depletion of the vascular compartment through renal and hormonal adjustments in sodium and osmolality, and body mass losses of at least $2 \%$ to $3 \%$ during prolonged exercise may be necessary to maintain euhydration.

\section{Excessive concern about hypohydration?}

In our examination of the literature, we find no controlled, laboratory-based studies using exercise protocols mirroring real-world exercise conditions (ie, those in which athletes are free to alter exercise intensity according to perceived exertion and the knowledge of completed and uncompleted distance and time) showing that drinking-to-thirst-associated hypohydration of at least $2 \%$ of body mass impedes running ${ }^{9,10}$ or cycling ${ }^{11}$ performance, compared with a scheduled rate of fluid 
intake aimed at preventing loss in body mass. What these studies collectively show is that thirst-driven fluid intake induces no performance disadvantage compared with programmed fluid intake, although it is evident that drinking less than what is dictated by thirst impairs endurance performance. ${ }^{11,12}$ Furthermore, a metaanalysis found that hypohydration up to $4 \%$ of body mass did not impair endurance performance during realworld exercise conditions. ${ }^{13}$

So, why is it that there is a widespread belief that hypohydration of at least $2 \%$ of body mass impairs performance? We believe it is because those controlledlaboratory studies arriving at such conclusions used methodologies that render them invalid in determining the real impact of hypohydration during outdoors conditions. ${ }^{14}$ In fact, in addition to preventing athletes from drinking before or during exercise, most studies have failed to provide appropriate convective cooling or blinded subjects from the hydration treatment received. When studies are designed so that subjects are allowed to quench their thirst during exercise, receive adequate ventilation, or are blinded to the experimental conditions, hypohydration greater than $2 \%$ has systematically been demonstrated not to impair performance or result in serious elevations in core temperature. $^{7,9,11}$

It is also noteworthy that it is quite common for observational field studies to report significant negative correlations between body mass loss and performance time. In other words, those with the greatest loss in body mass tend to be the fastest. The fastest long-distance triathletes, ${ }^{15}$ marathoners, ${ }^{16}$ and ultramarathoners ${ }^{17}$ often complete races with body mass losses of $4 \%$ to $9 \%$. Thus, mass losses in excess of $2 \%$ to $3 \%$ during prolonged exercise are well tolerated by elite athletes, but optimal hydration levels remain unknown.

With regard to exercise-associated muscle cramping, growing evidence from experimental ${ }^{18}$ and cohort $^{19}$ studies indicates that cramping results from neurologic changes rather than uncompensated water and sodium losses incurred during exercise. A lack of difference between those with and without cramping in postrace body mass change, plasma sodium concentration, and sodium intake provides further evidence that exerciseassociated muscle cramping is not related to fluid and sodium imbalances. ${ }^{20}$

\section{Thirst As A Stimulus To Maintain Appropriate Hydration}

The sensation of thirst in animals is a behavioral urge, driven largely by physiologic mediators that are activated when total body water content is low and antidiuresis is maximal. The 2 main sensors that detect physiologically relevant decreases in body water are the osmoreceptors located within the hypothalamus and baroreceptors located within the cardiac atria. Small $(1 \%-2 \%)$ elevations in the effective osmotic pressure of plasma can stimulate central osmoreceptors and trigger thirst. ${ }^{21}$ Thirst generally occurs when plasma osmolality exceeds $288 \mathrm{mOsmol} / \mathrm{kg} \mathrm{H}_{2} \mathrm{O},{ }^{22}$ which is still within the normal physiological range of 275 to 295 $\mathrm{mOsmol} / \mathrm{kg} \mathrm{H}_{2} \mathrm{O}$, although wide individual variation exists with regard to osmotic stimulation thresholds for both thirst and arginine vasopressin release. ${ }^{23}$ Peripheral baroreceptors stimulate thirst when circulating plasma volume decreases by more than $10 \%$ to $15 \%$. $^{22}$ Thus, thirst is stimulated to bring water into the body to either dilute rising blood solute concentrations or restore plasma volume, or both.

Subjects hypohydrated by performing light exercise have demonstrated that $65 \%$ of the variance in fluid intake was accounted for by changes in plasma osmolality or volume. ${ }^{24}$ Thus, factors other than thirst likely contribute to fluid intake, including oropharyngeal factors, mouth state, stomach fullness, beverage temperature, competing homeostatic mechanisms (ie, hunger, blood glucose), psychological factors, and situational factors including ambient temperature, exercise context, and duration. Older humans are also thought to have a lower thirst sensitivity to hypertonicity compared with younger individuals. ${ }^{25}$

Nevertheless, laboratory trials have demonstrated that drinking according to thirst is adequate to avoid body mass losses greater than $2 \%$ during low to moderate intensity exercise in the heat, as well as attenuate thermal and circulatory strain. ${ }^{26}$ Field studies have also shown that drinking to thirst, even during prolonged exercise up to 30 hours under hot ambient conditions, will allow maintenance of what we consider to be proper hydration when considering mass loss from stored fuel. ${ }^{5,6,27}$ Even when athletes begin aerobic exercise hypohydrated, the drive to drink during exercise is substantially magnified to optimally regulate plasma volume and osmolality ${ }^{28}$ and endurance performance. ${ }^{29}$

In contrast to drinking according to thirst, forced fluid replacement during exercise has been shown to enhance gastrointestinal distress. ${ }^{9,30}$ If severe enough, gastrointestinal-related problems may impede endurance performance. $^{30}$

\section{Problems With Alternative Methods For Maintaining Proper Hydration}

Various alternative methods for maintaining proper hydration during exercise have been suggested, but each 
may be impractical, unnecessary, invalid, or potentially dangerous depending on the circumstances. Perhaps the most commonly recommended method is based on a calculated sweat rate from body mass change during a known duration of exercise. This method is subject to the myriad of personal, exercise-related, clothing, and environmental factors that dynamically affect sweating. Small errors in estimating sweat rate could precipitate major imbalances in fluid homeostasis during prolonged exercise. For example, an overestimate of only $100 \mathrm{~mL} / \mathrm{h}$ during a 161-km ultramarathon could result in a fluid overload of $3 \mathrm{~L}$ when urine production is suppressed by nonosmotic arginine vasopressin secretion, a volume that would be adequate to induce EAH.

Plasma osmolality is the criterion (nonbehavioral) index of hydration status. It is physiologically regulated and functionally important and has a high sensitivity for functional hypohydration. But it is usually impractical to monitor and is one-dimensional. Urine-based measures, such as color, osmolality, specific gravity, production rate, and micturition frequency, are convenient but also reflect fluid and nonfluid-regulatory influences on renal function and are thus subject to problems of sensitivity and specificity (eg, confounding of urine color by vitamin supplementation or rhabdomyolysis, urine volume reduction that could be present with EAH despite overhydration). As such, thirst appears to be the most viable method for proper hydration given the disparate circumstances under which humans perform.

\section{Conclusions}

In the preceding paragraphs, we have offered scientific support for our premise that 1) some mass loss is appropriate during exercise, 2) exercise performance is not necessarily impaired nor is the risk of heat illness necessarily increased with modest mass loss, 3 ) drinking to thirst will generally be adequate to maintain hydration levels within a few percent of body mass loss, and 4) methods of maintaining hydration besides drinking to thirst are fraught with problems. The very few studies directly comparing prescribed vs self-determined drinking support our case. Nevertheless, even without considering the scientific evidence related to this essential question, one would have to believe it remarkable that the human species would exist if thirst were inadequate to guide drinking during exercise.

\section{References}

1. Sawka MN, Burke LM, Eichner ER, Maughan RJ, Montain SJ, Stachenfeld NS. American College of Sports Medicine. American College of Sports Medicine position stand. Exercise and fluid replacement. Med Sci Sports Exerc. 2007;39:377-390.

2. Casa DJ, Armstrong LE, Hillman SK, et al. National Athletic Trainers' Association position statement: Fluid replacement for athletes. J Athl Train. 2000;35:212-224.

3. Hew-Butler T, Rosner MH, Fowkes-Godek S, et al. Statement of the Third International Exercise-Associated Hyponatremia Consensus Development Conference, Carlsbad, California, 2015. Clin J Sport Med. 2015;25:303320.

4. Olsson KE, Saltin B. Variation in total body water with muscle glycogen changes in man. Acta Physiol Scand. 1970;80:11-18.

5. Tam N, Nolte HW, Noakes TD. Changes in total body water content during running races of $21.1 \mathrm{~km}$ and $56 \mathrm{~km}$ in athletes drinking ad libitum. Clin J Sport Med. 2011;21:218-225.

6. Nolte HW, Noakes TD, van Vuuren B. Protection of total body water content and absence of hyperthermia despite $2 \%$ body mass loss ('voluntary dehydration') in soldiers drinking ad libitum during prolonged exercise in cool environmental conditions. Br J Sports Med. 2011;45:1106-1112.

7. Wall BA, Watson G, Peiffer JJ, Abbiss CR, Siegel R, Laursen PB. Current hydration guidelines are erroneous: dehydration does not impair exercise performance in the heat. Br J Sports Med. 2015;49:1077-1083.

8. Saltin B. Circulatory response to submaximal and maximal exercise after thermal dehydration. J Appl Physiol. 1964; 19:1125-1132.

9. Dion T, Savoie FA, Asselin A, Gariepy C, Goulet ED. Half-marathon running performance is not improved by a rate of fluid intake above that dictated by thirst sensation in trained distance runners. Eur J Appl Physiol. 2013;113: 3011-3020.

10. Lee MJ, Hammond KM, Vasdev A, et al. Self-selecting fluid intake while maintaining high carbohydrate availability does not impair half-marathon performance. Int $J$ Sports Med. 2014;35:1216-1222.

11. Dugas JP, Oosthuizen U, Tucker R, Noakes TD. Rates of fluid ingestion alter pacing but not thermoregulatory responses during prolonged exercise in hot and humid conditions with appropriate convective cooling. Eur J Appl Physiol. 2009;105:69-80.

12. Goulet ED. Effect of exercise-induced dehydration on time-trial exercise performance: a meta-analysis. $\mathrm{Br} J$ Sports Med. 2011;45:1149-1156.

13. Goulet ED. Effect of exercise-induced dehydration on endurance performance: evaluating the impact of exercise protocols on outcomes using a meta-analytic procedure. $\mathrm{Br}$ J Sports Med. 2013;47:679-686.

14. Cotter JD, Thornton SN, Lee JK, Laursen PB. Are we being drowned in hydration advice? Thirsty for more? Extrem Physiol Med. 2014 Oct 29;3:18, http://dx.doi.org/ 10.1186/2046-7648-3-18.

15. Sharwood KA, Collins M, Goedecke JH, Wilson G, Noakes TD. Weight changes, medical complications, and 
performance during an Ironman triathlon. Br J Sports Med. 2004;38:718-724.

16. Zouhal H, Groussard C, Minter G, et al. Inverse relationship between percentage body weight change and finishing time in 643 forty-two-kilometre marathon runners. $\mathrm{Br} \mathrm{J}$ Sports Med. 2011;45:1101-1105.

17. Hoffman MD, Hew-Butler T, Stuempfle KJ. Exerciseassociated hyponatremia and hydration status in 161-km ultramarathoners. Med Sci Sports Exerc. 2013;45: 784-791.

18. Miller KC, Mack GW, Knight KL, et al. Three percent hypohydration does not affect threshold frequency of electrically induced cramps. Med Sci Sports Exerc. 2010;42:2056-2063.

19. Schwellnus MP, Allie S, Derman W, Collins M. Increased running speed and pre-race muscle damage as risk factors for exercise-associated muscle cramps in a $56 \mathrm{~km}$ ultramarathon: a prospective cohort study. Br J Sports Med. 2011;45:1132-1136.

20. Hoffman MD, Stuempfle KJ. Muscle cramping during a 161-km ultramarathon: comparison of characteristics of those with and without cramping. Sports Med Open 2015;1:8.

21. McKinley MJ, Johnson AK. The physiological regulation of thirst and fluid intake. News Physiol Sci. 2004;19:1-6.

22. Verbalis JG. Disorders of body water homeostasis. Best Pract Res Clin Endocrinol Metab. 2003;17:471-503.

23. Robertson GL. Abnormalities of thirst regulation. Kidney Int. 1984;25:460-469.

24. Engell DB, Maller O, Sawka MN, Francesconi RN, Drolet L, Young AJ. Thirst and fluid intake following graded hypohydration levels in humans. Physiol Behav. 1987;40: 229-236.

25. Phillips PA, Bretherton M, Johnston CI, Gray L. Reduced osmotic thirst in healthy elderly men. Am J Physiol. 1991; 261(1 Pt 2):R166-R171.

26. Armstrong LE, Maresh CM, Gabaree CV, et al. Thermal and circulatory responses during exercise: effects of hypohydration, dehydration, and water intake. J Appl Physiol. 1997;82:2028-2035.

27. Hoffman MD, Stuempfle KJ. Is sodium supplementation necessary to avoid dehydration during prolonged exercise in the heat? J Strength Cond Res. 2016;30:615-620.

28. Maresh CM, Gabaree-Boulant CL, Armstrong LE, et al. Effect of hydration status on thirst, drinking, and related hormonal responses during low-intensity exercise in the heat. J Appl Physiol. 2004;97:39-44.

29. Berkulo MA, Bol S, Levels K, Lamberts RP, Daanen HA, Noakes TD. Ad-libitum drinking and performance during a 40-km cycling time trial in the heat. Eur J Sport Sci. 2016;15:213-220.

30. Daries HN, Noakes TD, Dennis SC. Effect of fluid intake volume on 2-h running performances in a 25 degrees $\mathrm{C}$ environment. Med Sci Sports Exerc. 2000;32:1783-1789.
COUNTERVIEW: Is Drinking to Thirst Adequate to Appropriately Maintain Hydration Status During Prolonged Endurance Exercise? No

Lawrence E. Armstrong, PhD; Evan C. Johnson, PhD; Michael F. Bergeron, PhD

From the Department of Kinesiology, Human Performance Laboratory, Unit 1110, University of Connecticut, Storrs, CT (Dr Armstrong); the Division of Kinesiology and Health, Human Integrated Physiology Laboratory, University of Wyoming, Laramie, WY (Dr Johnson); and the Youth Sports of the Americas, Birmingham, AL (Dr Bergeron).

No, drinking to thirst (DTT) is not adequate to maintain hydration status or optimal performance during prolonged endurance exercise (which should not be limited to timed events). Enough individual and athletic scenarios exist that preclude the DTT recommendation from being an absolute, unwavering, universally effective guideline suitable for all participants. Importantly, "drinking to thirst" is not equivalent to ad libitum fluid intake, ${ }^{1}$ and we are certainly not supporting drinking in excess or "as much as tolerable" during or after exercise. However, optimal fluid intake during any prolonged, repeated, or intermittent physical activity is indeed situation- and individual-specific, which can partially rely on thirst as part of an evidence-informed advancedplanned fluid intake strategy.

To further elucidate, we begin by defining terms and underscoring the key relevant issues. The phrase appropriately maintain hydration may be applied in terms of preventing fluid overload, or from the perspective of optimally supporting training-competitive athletic performance. Specific to fluid overload (ie, intake and retention of water exceeding the rate of fluid loss), the concept of DTT (ie, relying solely on one's personal

Drs. Armstrong and Bergeron have no pertinent disclosures or conflicts of interest.

Dr. Johnson discloses that he is currently funded for research by Danone Research, Palaiseau, France.

Corresponding author: Lawrence E. Armstrong, $\mathrm{PhD}$, University of Connecticut, Department of Kinesiology, Human Performance Laboratory, Unit 1110, Storrs, CT 06029-1110 (e-mail: lawrence.armstrong@ uconn.edu). 